\title{
Comment on: Frequency and Associated Costs of Anaphylaxis- and Hypersensitivity-Related Adverse Events for Intravenous Iron Products in the USA: An Analysis Using the US Food and Drug Administration Adverse Event Reporting System
}

\author{
Michael Auerbach ${ }^{1}$
}

Accepted: 15 February 2021 / Published online: 9 April 2021

(c) The Author(s), under exclusive licence to Springer Nature Switzerland AG part of Springer Nature 2021

\section{Dear Editor,}

Having read the article by Trumbo et al. [1], published in Drug Safety, I wish to express several serious concerns. While this article was interesting, the authors used a methodology specifically proscribed by the US FDA. This methodology is also referenced in Wysowski et al. [2], the authors of which are all from the FDA and concluded not only that "allergic reactions are possible with all four parenteral iron products" but also that the current system of spontaneous adverse event reporting, particularly in the absence of headto-head trials, does not allow the relative rates of serious adverse events (SAEs) to be determined. Given this strong conclusion, it is prudent to look at the available head-to-head trials and comment on one of the studies cited by Trumbo et al. [1], that of Wang et al. [3] published in JAMA.

The FIRM trial by Adkinson et al. [4], also referenced in Trumbo et al. [1], used a double-blind prospective paradigm and examined 1997 patients who received two doses of either ferumoxytol or ferric carboxymaltose (FCM) according to the FDA label. No differences in SAEs were found. Trumbo et al. [1], funded by the manufacturer of FCM, failed to mention prospective comparisons of FCM and iron sucrose, ferumoxytol and iron sucrose, ferric derisomaltose (formerly iron isomaltoside) and iron sucrose, or ferric derisomaltose and FCM [5-8]. None of these studies reported

This comment refers to the article available online at https://doi. org/10.1007/s40264-020-01022-2.

An author's reply to this commentary available at https://doi. org/10.1007/s40264-021-01059-x.

Michael Auerbach

mauerbachmd@abhemonc.com

1 Auerbach Hematology-Oncology, Baltimore, MD, USA any differences in adverse events. Nor did they mention the study by Wolf et al. [8], published in JAMA, which reported a much higher incidence of significant hypophosphatemia with FCM than with derisomaltose. The authors also omitted a recently published meta-analysis of the prospective randomized trials, which reported no difference in adverse events among the "dextran-derived" formulations and FCM and iron sucrose, effectively mitigating benefit from the inappropriate use of the term "dextran derived."

In Tables e 3 and e 4 in the online supplementary material for Wang et al. [3], iron dextran had the lowest risk of death within $24 \mathrm{~h}$ of administration of any iron formulation, effectively contradicting their own conclusions [10]. Further, during the study, the only truly dangerous iron formulation, high-molecular-weight (HMW) iron dextran, was still available, and the authors did not distinguish between this now unavailable HMW formulation and the much safer lowmolecular-weight formulation. Given these data, the Wang et al. [3] paper should not be used to support the relative safety of any product over another.

I have previously reviewed, under the Freedom of Information Act, thousands of spontaneous AE reports submitted to the FDA over a 10-year period [11]. It was impossible to glean which patients received inappropriate premedication with diphenhydramine or even more inappropriate interventions with vasopressors or antihistamines, all of which convert self-limited minor infusion reactions to hemodynamically significant SAEs, which are then ostensibly attributed to the intravenous iron.

Considering the burgeoning use of intravenous iron in a litany of maladies in which oral iron is either ineffective or harmful, I believe the conclusions in Trumbo et al. [1] are inaccurate, inconsistent with the overwhelming preponderance of prospective randomized published evidence, misrepresentative, and harmful to the field. Of concern is that 
the study was funded by the company with the most to gain from these authors' conclusions.

\section{Declarations}

Conflict of Interest Michael Auerbach has received research funding from AMAG Pharmaceuticals for data management only and has given educational, non-promotional programs for Pharmacosmos and Pfizer. The author has no financial interest in any intravenous iron formulation.

Author contributions Michael Auerbach wrote this article in its entirety and read and approved the final version.

Funding Not applicable.

Ethics approval Not applicable.

Consent to participate Not applicable.

Consent for publication Not applicable.

Availability of data Not applicable.

Code Availability Not applicable.

\section{References}

1. Trumbo H, Kaluza K, Numan S, et al. Frequency and associated costs of anaphylaxis and hypersensitivity-related adverse events for intravenous iron products in the USA: An analysis using the US Food and Drug Administration Adverse Event Reporting System. Drug Saf. 2021;44(1):107-19. https://doi.org/10.1007/s4026 4-020-01022-2.
2. Wysowski D, Swartz L, Borders-Hemphill V, et al. Use of parenteral iron products and serious anaphylactic-type reactions. Am J Hematol. 2010;85:650-4.

3. Wang C, Graham D, Kane R, et al. Comparative risk of anaphylactic reactions associated with intravenous iron products. JAMA. 2015;314:2062-8.

4. Adkinson N, Strauss W, Macdougall I, et al. Comparative safety of intravenous ferumoxytol versus ferric carboxymaltose in iron deficiency anemia: a randomized trial. Am J Hematol. 2018;93:683-90.

5. Onken J, Bregman D, Harrington R, et al. Ferric carboxymaltose in patients with iron-deficiency anemia and impaired renal function: the REPAIR-IDA trial. Nephrol Dial Transplant. 2014;29:833-42.

6. Macdougall I, Strauss W, McLaughlin J, et al. A randomized comparison of ferumoxytol and iron sucrose for treating iron deficiency anemia in patients with CKD. Clin J Am Soc Nephrol 9:705-712.

7. Auerbach M, Henry D, Derman R, et al. A prospective, multicenter, randomized comparison of iron isomaltoside versus iron sucrose to determine relative hemoglobin concentration increment and improvement in quality of life parameters in patients with iron deficiency anemia. Am J Hematol. 2019;94:1007-14.

8. Wolf M, Rubin J, Achebe M, et al. Effects of iron isomaltoside vs ferric carboxymaltose on hypophosphatemia in iron-deficiency anemia. JAMA. 2020;323:432-43.

9. Achebe M, DeLoughery T. Clinical data for intravenous irondebunking the hype around hypersensitivity. Transfusion. 2020;60:1154-9.

10. DeLoughery T, Auerbach M. Is low-molecular weight iron dextran really the most risky iron?--Unconvincing data from an unconvincing study. Am J Hematol. 2016;91:451-2.

11. Auerbach M, Ballard H. Clinical use of intravenous iron: administration, efficacy and safety. In Hematology 2010. American Society of Hematology Education Program Book: 338-347. Washington DC, December 2010. 